\title{
On the Classical Paranormed Sequence Spaces and Related Duals over the Non-Newtonian Complex Field
}

\author{
Uğur Kadak, ${ }^{1}$ Murat Kirişci, ${ }^{2}$ and Ahmet Faruk Çakmak ${ }^{3}$ \\ ${ }^{1}$ Department of Mathematics, Faculty of Sciences and Arts, Bozok University, 66200 Yozgat, Turkey \\ ${ }^{2}$ Department of Mathematical Education, Hasan Ali Yücel Education Faculty, Istanbul University, 34470 Istanbul, Turkey \\ ${ }^{3}$ Department of Mathematical Engineering, Yildiz Technical University, 80750 Istanbul, Turkey \\ Correspondence should be addressed to Uğur Kadak; ugurkadak@gmail.com
}

Received 20 October 2014; Accepted 7 May 2015

Academic Editor: Jeff Connor

Copyright (C) 2015 Uğur Kadak et al. This is an open access article distributed under the Creative Commons Attribution License, which permits unrestricted use, distribution, and reproduction in any medium, provided the original work is properly cited.

\begin{abstract}
The studies on sequence spaces were extended by using the notion of associated multiplier sequences. A multiplier sequence can be used to accelerate the convergence of the sequences in some spaces. In some sense, it can be viewed as a catalyst, which is used to accelerate the process of chemical reaction. Sometimes the associated multiplier sequence delays the rate of convergence of a sequence. In the present paper, the classical paranormed sequence spaces have been introduced and proved that the spaces are $\star$-complete. By using the notion of multiplier sequence, the $\alpha-, \beta$-, and $\gamma$-duals of certain paranormed spaces have been computed and their basis has been constructed.
\end{abstract}

\section{Introduction}

The theory of sequence spaces is the fundamental of summability. Summability is a wide field of mathematics, mainly in analysis and functional analysis, and has many applications, for instance in numerical analysis to speed up the rate of convergence, operator theory, the theory of orthogonal series, and approximation theory. The classical summability theory deals with the generalization of the convergence of sequences or series of real or complex numbers. Besides this, the studies on paranormed sequence spaces were initiated by Nakano [1] and Simons [2] at the initial stage. Later on it was further studied by Maddox [3], Lascarides [4], and Lascarides and Maddox [5]. In recent years, Mursaleen et al. [6-8] have investigated some matrix transformations of paranormed sequence spaces. Also Kiriş̧̧i and Başar [9, 10] motivated the notion of generalized difference matrix and Demiriz and Çakan [11] determined some new paranormed sequence spaces.

In the period from 1967 till 1972, Grossman and Katz [12] introduced the non-Newtonian calculus consisting of the branches of geometric, bigeometric, quadratic, biquadratic calculus, and so forth. Also Grossman extended this notion to the other fields in $[13,14]$. All these calculi can be described simultaneously within the framework of a general theory. We prefer to use the name non-Newtonian to indicate any of the calculi other than the classical calculus. Every property in classical calculus has an analogue in non-Newtonian calculus which is a methodology that allows one to have a different look at problems which can be investigated via calculus. In some cases, for example for wage-rate (in dollars, euro, etc.) related problems, the use of bigeometric calculus which is a kind of non-Newtonian calculus is advocated instead of a traditional Newtonian one.

Bashirov et al. $[15,16]$ have recently concentrated on nonNewtonian calculus and gave the results with applications corresponding to the well-known properties of derivatives and integrals in classical calculus. Further Misirli and Gurefe have introduced multiplicative Adams Bashforth-Moulton method for numerical solution of differential equations in [17]. Also some authors have also worked on classical sequence spaces and related topics by using non-Newtonian calculus [18, 19]. Further Kadak [20] and Kadak et al. [21-23] have determined Kothe-Toeplitz duals and matrix 
transformations between certain sequence spaces over the non-Newtonian complex field and have generalized RungeKutta method with respect to the non-Newtonian calculus.

\section{Preliminaries, Background, and Notations}

A generator is a one-to-one function whose domain is $\mathbb{R}$ and whose range is a subset $\mathbb{R}_{\alpha}$ of $\mathbb{R}$ where $\mathbb{R}_{\alpha}:=\{\alpha\{x\}$ : $x \in \mathbb{R}\}$. Each generator generates exactly one arithmetic, and conversely each arithmetic is generated by exactly one generator. For example, the identity function $I$ generates classical arithmetic, and exponential function generates geometric (multiplicative) arithmetic. As a generator, we choose the function $\alpha$ such that those basic algebraic operations are defined as follows:

$$
\begin{aligned}
& \alpha \text {-addition } x+y=\alpha\left\{\alpha^{-1}(x)+\alpha^{-1}(y)\right\} \\
& \alpha \text {-subtraction } x-y=\alpha\left\{\alpha^{-1}(x)-\alpha^{-1}(y)\right\} \\
& \alpha \text { - multiplication } x \dot{x} y=\alpha\left\{\alpha^{-1}(x) \times \alpha^{-1}(y)\right\} \\
& \alpha \text { - division } \frac{x}{y}=\alpha\left\{\alpha^{-1}(x) \div \alpha^{-1}(y)\right\} \\
& \alpha \text { - order } x \dot{<} y \Longleftrightarrow \alpha^{-1}(x)<\alpha^{-1}(y)
\end{aligned}
$$

for all $x, y \in \mathbb{R}_{\alpha} \subseteq \mathbb{R}$. As an example if we choose the function $\alpha=$ exp:

$$
\begin{aligned}
\alpha: \mathbb{R} & \longrightarrow \mathbb{R}_{\exp } \subseteq \mathbb{R} \\
x & \longmapsto y=\alpha(x)=e^{x},
\end{aligned}
$$

$\alpha$-arithmetic can be interpreted as geometric arithmetic:

$$
\begin{aligned}
& \alpha \text {-addition } x+y=e^{\{\ln x+\ln y\}}=x \cdot y \\
& \alpha \text { - subtraction } x-y=e^{\{\ln x-\ln y\}}=x \div y \\
& \alpha \text { - multiplication } x \dot{x} y=e^{\{\ln x \ln y\}}=x^{\ln y}=y^{\ln x} \\
& \alpha \text { - division } \frac{x}{y} \cdot=e^{\{\ln x / \ln y\}}=x^{1 / \ln y} .
\end{aligned}
$$

By an arithmetic, we mean a complete ordered field whose realm is a subset of $\mathbb{R}$. There are infinitely many arithmetics, all of which are isomorphic, that is, structurally equivalent. The $\alpha$-positive real numbers, denoted by $\mathbb{R}_{\alpha}^{+}$, are the numbers $x$ in $\mathbb{R}_{\alpha}$ such that $\dot{0}<x$; the $\alpha$-negative real numbers, denoted by $\mathbb{R}_{\alpha}^{-}$, are those for which $x<\dot{0}$. The $\alpha$-zero, $\dot{0}$, and the alpha-one, $\mathrm{i}$, turn out to be $\alpha(0)$ and $\alpha(1)$. Also $\alpha(-p)=\alpha\left\{-\alpha^{-1}(\dot{p})\right\}=\dot{-} \dot{p}$ holds for all $p \in \mathbb{Z}^{+}$. Thus the set of all $\alpha$-integers can be given by

$$
\begin{aligned}
\mathbb{Z}_{\alpha} & =\{\ldots, \alpha(-2), \alpha(-1), \alpha(0), \alpha(1), \alpha(2), \ldots\} \\
& =\{\ldots, \dot{-} \dot{2}, \dot{-} \dot{i}, \dot{0}, \dot{1}, \dot{2}, \ldots\} .
\end{aligned}
$$

One can immediately conclude that the set of exp-integer can be written as

$$
\mathbb{Z}_{\exp }=\left\{\ldots, \frac{1}{e^{2}}, \frac{1}{e}, 1, e, e^{2}, \ldots\right\} .
$$

Besides, the $\alpha$-summation is defined by

$$
\begin{aligned}
\alpha \sum_{k=0}^{\infty} x_{k} & =\alpha\left\{\sum_{k=0}^{\infty} \alpha^{-1}\left(x_{k}\right)\right\} \\
& =\alpha\left\{\alpha^{-1}\left(x_{0}\right)+\cdots+\alpha^{-1}\left(x_{k}\right)+\cdots\right\}
\end{aligned}
$$

for all $x_{k} \in \mathbb{R}_{\alpha} \subseteq \mathbb{R}$.

Definition 1 (see [18]). Let $X$ be a nonempty set and $d_{\alpha}: X \times$ $X \rightarrow \mathbb{R}_{\alpha} \subseteq \mathbb{R}$ be a function such that, for all $x, y, z \in X$, the following axioms hold:

$$
\begin{aligned}
& \text { (NM1) } d_{\alpha}(x, y)=\dot{0} \text { if and only if } x=y, \\
& \text { (NM2) } d_{\alpha}(x, y)=d_{\alpha}(y, x), \\
& \text { (NM3) } d_{\alpha}(x, y) \leq d_{\alpha}(x, z) \dot{+} d_{\alpha}(z, y) .
\end{aligned}
$$

Then, the pair $\left(X, d_{\alpha}\right)$ and $d_{\alpha}$ are called an $\alpha$-metric space and an $\alpha$-metric on $X$, respectively.

Throughout this paper, we define the $p$ th $\alpha$-exponent $x^{p_{\alpha}}$ and $q$ th $\alpha$-root $x^{(1 / q)_{\alpha}}$ of $x \in \mathbb{R}_{\alpha} \subseteq \mathbb{R}$ as

$$
\begin{aligned}
x^{2 \alpha} & =x \dot{\times} x=\alpha\left\{\alpha^{-1}(x) \times \alpha^{-1}(x)\right\}=\alpha\left\{\left[\alpha^{-1}(x)\right]^{2}\right\} \\
x^{3 \alpha} & =x^{2 \alpha} \dot{\times} x \\
& =\alpha\left\{\alpha^{-1}\left\{\alpha\left[\alpha^{-1}(x) \times \alpha^{-1}(x)\right]\right\} \times \alpha^{-1}(x)\right\} \\
& =\alpha\left\{\left[\alpha^{-1}(x)\right]^{3}\right\}
\end{aligned}
$$

$$
x^{p_{\alpha}}=x^{(p-1)_{\alpha}} \dot{\times} x=\alpha\left\{\left[\alpha^{-1}(x)\right]^{p}\right\} .
$$

Hence $\sqrt[\alpha]{x}=x^{(1 / 2)_{\alpha}}=y$ provided there exists an $y \in \mathbb{R}_{\alpha} \subseteq \mathbb{R}$ such that $y^{2_{\alpha}}=x$. For each $\alpha$-nonnegative number $x$, the symbol $\sqrt[\alpha]{x}$ will be used to denote $\alpha\left\{\left[\alpha^{-1}(x)\right]^{1 / 2}\right\}$ which is the unique $\alpha$-nonnegative number $y$ whose $\alpha$-square is equal to $x$. For each number $x \in \mathbb{R}_{\alpha}, \sqrt[\alpha]{x^{2} \alpha}=|x|_{\alpha}=\alpha\left(\left|\alpha^{-1}(x)\right|\right)$ where the absolute value $|x|_{\alpha}$ of $x \in \mathbb{R}_{\alpha}$ is defined by

$$
|x|_{\alpha}= \begin{cases}x, & x>0 \\ \dot{0}, & x=0 \\ -x, & x<0\end{cases}
$$

Definition 2 (see [18]). Let $X=\left(X, d_{\alpha}\right)$ be an $\alpha$-metric space. Then the basic notions can be defined as follows.

(a) A sequence $x=\left(x_{k}\right)$ is a function from the set $\mathbb{N}$ into the set $\mathbb{R}_{\alpha}$. The $\alpha$-real number $x_{k}$ denotes the value of the function at $k \in \mathbb{N}$ and is called the $k$ th term of the sequence.

(b) A sequence $\left(x_{n}\right)$ in $X=\left(X, d_{\alpha}\right)$ is said to be $\alpha$ convergent if, for every given $\varepsilon>\dot{0}\left(\varepsilon \in \mathbb{R}_{\alpha}\right)$, there 
exist an $n_{0}=n_{0}(\varepsilon) \in \mathbb{N}$ and $x \in X$ such that $d_{\alpha}\left(x_{n}, x\right)=\left|x_{n}-x\right|_{\alpha} \dot{<} \varepsilon$ for all $n>n_{0}$ which is denoted by ${ }^{\alpha} \lim _{n \rightarrow \infty} x_{n}=x$ or $x_{n} \stackrel{\alpha}{\rightarrow} x$, as $n \rightarrow \infty$.

(c) A sequence $\left(x_{n}\right)$ in $X=\left(X, d_{\alpha}\right)$ is said to be $\alpha$-Cauchy if for every $\varepsilon>0$ there is an $n_{0}=n_{0}(\varepsilon) \in \mathbb{N}$ such that $d_{\alpha}\left(x_{n}, x_{m}\right) \dot{<}$ for all $m, n>n_{0}$.

Following [12], we give a new type of calculus by using the notion of non-Newtonian complex numbers, denoted by $\star$-calculus ("star-"), which is a branch of non-Newtonian calculus. From now on we will use the notation $\star$-calculus corresponding calculus which is based on two arbitrarily selected generator functions.

2.1. $\star$-Arithmetic ("Star"-Arithmetic). Suppose that $\alpha$ and $\beta$ are two arbitrarily selected generators and ("star-") also is the ordered pair of arithmetics, that is, $\beta$-arith-metic and $\alpha$ arithmetic. The sets $\left(\mathbb{R}_{\beta}, \ddot{+}, \ddot{-}, \ddot{x}, \ddot{j}\right)$ and $\left(\mathbb{R}_{\alpha}, \dot{+}, \dot{-}, \dot{x}, \dot{j}\right)$ are complete ordered fields (see [19]) and beta- (alpha-) generator generates beta- (alpha-) arithmetics, respectively. Definitions given for $\beta$-arithmetic are also valid for $\alpha$ arithmetic. The important point to note here is that $\alpha$ arithmetic is used for arguments and $\beta$-arithmetic is used for values; in particular, changes in arguments and values are measured by $\alpha$-differences and $\beta$-differences, respectively.

Definition 3 (see [13]). The $\star$-limit of a function $f$ at an element $a$ in $\mathbb{R}_{\alpha}$ is, if it exists, the unique number $b$ in $\mathbb{R}_{\beta}$ such that

$$
\begin{aligned}
& { }^{\star} \lim _{x \rightarrow a} f(x)=b \\
& \Longleftrightarrow \forall \varepsilon \ddot{>0}, \exists \delta>\dot{0} \ni|f(x) \ddot{-} b|_{\beta} \ddot{<} \varepsilon \forall x \in \mathbb{R}_{\alpha},|x \dot{-} a|_{\alpha}<\delta
\end{aligned}
$$

and is denoted by ${ }^{\star} \lim _{x \rightarrow a} f(x)=b$. Also we can give the definition for every sequence $\left(x_{n}\right)$ of arguments of $f$ distinct from $a$; if $\left(x_{n}\right)$ is $\alpha$-convergent to $a$, then $\left\{f\left(x_{n}\right)\right\} \beta$-converges to $b$.

A function $f$ is $\star$-continuous at a point $a$ in $\mathbb{R}_{\alpha}$ if and only if $a$ is an argument of $f$ and ${ }^{\star} \lim _{x \rightarrow a} f(x)=f(a)$. When $\alpha$ and $\beta$ are the identity function $I$, the concepts of $\star$-limit and $\star$-continuity are identical with those of classical limit and classical continuity.

The isomorphism from $\alpha$-arithmetic to $\beta$-arithmetic is the unique function $\iota$ (iota) that possesses the following three properties.

(i) $\iota$ is one to one.

(ii) $\iota$ is from $\mathbb{R}_{\alpha}$ to $\mathbb{R}_{\beta}$.

(iii) For any numbers $u$ and $v$ in $\mathbb{R}_{\alpha}$,

$$
\begin{aligned}
& \iota(u+v)=\iota(u) \ddot{+} \iota(v), \\
& \iota(u \dot{-} v)=\iota(u) \ddot{-} \iota(v),
\end{aligned}
$$

$$
\begin{aligned}
& \iota(u \dot{\times} v)=\iota(u) \ddot{\times} \iota(v), \\
& \iota(u j v)=\iota(u) \ddot{/} \iota(v) ;
\end{aligned}
$$$$
v \neq \dot{0}, u \leq v \Longleftrightarrow \iota(u) \ddot{\leq} \iota(v) .
$$

It turns out that $\iota(x)=\beta\left\{\alpha^{-1}(x)\right\}$ for every $x$ in $\mathbb{R}_{\alpha}$ and that $\iota(\dot{n})=\ddot{n}$ for every integer $n$. Since, for example, $u \dot{+} v=$ $\iota^{-1}\{\iota(u) \ddot{+} \iota(v)\}$, it should be clear that any statement in $\alpha$ arithmetic can readily be transformed into a statement in $\beta$ arithmetic.

2.2. Non-Newtonian Complex Field. Let $\dot{a} \in\left(\mathbb{R}_{\alpha}, \dot{+}, \dot{-}\right.$, $\dot{x}, \dot{l})$ and $\ddot{b} \in\left(\mathbb{R}_{\beta}, \ddot{+}, \ddot{-}, \ddot{x}, \ddot{l}\right)$ be arbitrarily chosen elements from corresponding arithmetics. Then the ordered pair $(\dot{a}, \ddot{b})$ is called $\mathrm{a} \star$-point and the set of all $\star$-points is called the set of $\star$-complex numbers, which is denoted by $\mathbb{C}^{\star}$; that is,

$$
\mathbb{C}^{\star}:=\left\{z^{\star}=(\dot{a}, \ddot{b}) \mid \dot{a} \in \mathbb{R}_{\alpha} \subseteq \mathbb{R}, \ddot{b} \in \mathbb{R}_{\beta} \subseteq \mathbb{R}\right\}
$$

Define the binary operations addition $(\oplus)$ and multiplication (๑) of $\star$-complex numbers $z_{1}^{\star}=\left(\dot{a}_{1}, \ddot{b}_{1}\right)$ and $z_{2}^{\star}=\left(\dot{a}_{2}, \ddot{b}_{2}\right)$ as

$$
\begin{aligned}
\oplus: \mathbb{C}^{\star} \times \mathbb{C}^{\star} & \longrightarrow \mathbb{C}^{\star} \\
\left(z_{1}^{\star}, z_{2}^{\star}\right) & \longmapsto z_{1}^{\star} \oplus z_{2}^{\star}=\left(\alpha\left\{a_{1}+a_{2}\right\}, \beta\left\{b_{1}+b_{2}\right\}\right) \\
& =\left(\dot{a}_{1}+\dot{a}_{2}, \ddot{b}_{1}+\ddot{b}_{2}\right) \\
\odot: \mathbb{C}^{\star} \times \mathbb{C}^{\star} & \longrightarrow \mathbb{C}^{\star} \\
\left(z_{1}^{\star}, z_{2}^{\star}\right) & \longmapsto z_{1}^{\star} \odot z_{2}^{\star} \\
& =\left(\alpha\left\{a_{1} a_{2}-b_{1} b_{2}\right\}, \beta\left\{a_{1} b_{2}+b_{1} a_{2}\right\}\right),
\end{aligned}
$$

where $\dot{a}_{1}, \dot{a}_{2} \in \mathbb{R}_{\alpha}$ and $\ddot{b}_{1}, \ddot{b}_{2} \in \mathbb{R}_{\beta}$.

Theorem 4 (see [19]). $\left(\mathbb{C}^{\star}, \oplus, \odot\right)$ is a field.

Following Grossman and Katz [12] we can give the definition of $\star$-distance regarding $\star$-calculus.

Definition 5 (see [19]). The $\star$-distance $d^{\star}$ between two arbitrarily elements $z_{1}^{\star}=\left(\dot{a}_{1}, \ddot{b}_{1}\right)$ and $z_{2}^{\star}=\left(\dot{a}_{2}, \ddot{b}_{2}\right)$ of the set $\mathbb{C}^{\star}$ is defined by

$$
\begin{aligned}
d^{\star}: \mathbb{C}^{\star} \times \mathbb{C}^{\star} & \longrightarrow[\ddot{0}, \infty)=B^{\prime} \subset \mathbb{R}_{\beta} \\
\left(z_{1}^{\star}, z_{2}^{\star}\right) & \longmapsto d^{\star}\left(z_{1}^{\star}, z_{2}^{\star}\right) \\
& =\left(\iota\left(\dot{a}_{1}-\dot{a}_{2}\right)^{2_{\alpha}} \ddot{+}\left(\ddot{b}_{1}-\ddot{b}_{2}\right)^{2_{\beta}}\right)^{(1 / 2)_{\beta}} \\
& =\beta\left\{\sqrt{\left(a_{1}-a_{2}\right)^{2}+\left(b_{1}-b_{2}\right)^{2}}\right\} .
\end{aligned}
$$


Definition 6 (see [20]). Given a sequence $\left(z_{k}^{\star}\right)=\left(\dot{x}_{k}, \ddot{y}_{k}\right)$ of $\star$-complex numbers, the formal notation

$$
\begin{aligned}
\star \sum_{k=0}^{\infty} z_{k}^{\star} & =z_{0}^{\star} \oplus z_{1}^{\star} \oplus z_{2}^{\star} \oplus \cdots \oplus z_{k}^{\star} \oplus \cdots \\
& =\left(\alpha \sum_{k=0}^{\infty} \dot{x}_{k}, \beta \sum_{k=0}^{\infty} \ddot{y}_{k}\right) \\
& =\left(\alpha\left\{\sum_{k=0}^{\infty} \alpha^{-1}\left\{x_{k}\right\}\right\}, \beta\left\{\sum_{k=0}^{\infty} \beta^{-1}\left\{x_{k}\right\}\right\}\right) \\
& \in \mathbb{C}^{\star},
\end{aligned}
$$

for all $k \in \mathbb{N}$, is called an infinite series with $\star$-complex terms or simply complex $\star$-series. Also, for integers $n \in \mathbb{N}$, the finite $\star$-sums $s_{n}^{\star}=\star \sum_{k=0}^{n} z_{k}^{\star}$ are called the partial sums of complex $\star$-series. If the sequence $\star$-converges to a complex number $s^{\star}$, then we say that the series $\star$-converges and write $s^{\star}=\sum_{n=0}^{\infty} z_{n}^{\star}$. The number $s^{\star}$ is then called the $\star$-sum of this series. If $\left(s_{n}\right) \star$-diverges, we say that the series $\star$-diverges or that it is $\star$-divergent.

Definition 7 (see [22]). Let $X$ be a real or complex linear space and let $\ddot{\|} \cdot \ddot{\|}$ be a function from $X$ to the set $\mathbb{R}_{\beta}^{+}$of nonnegative $\beta$-real numbers. Then the pair $(X, \ddot{\|} \cdot \ddot{\|})$ is called $a \star$-normed space and $\ddot{\|} \cdot \ddot{\|}$ is a $\star$-norm for $X$, if the following axioms are satisfied for all elements $x, y \in X$ and for all scalars $\lambda$ :

$$
\begin{aligned}
& (\mathrm{NN} 1) \ddot{\|} x \ddot{\|}=\ddot{0} \Leftrightarrow x=\theta^{\star}\left(\theta^{\star}=(\dot{0}, \ddot{0})\right), \\
& (\mathrm{NN} 2) \ddot{\|} \lambda \odot x \ddot{\|}=\ddot{\mid} \lambda \ddot{\mid} \ddot{\times} \ddot{\|} x \ddot{\|}, \\
& (\mathrm{NN} 3) \ddot{\|} x \oplus y\|\ddot{\leq} \ddot{\|} x \ddot{\|} \ddot{+} \ddot{\|} y\|
\end{aligned}
$$

It is trivial that a $\star$-norm $\ddot{\|} \cdot \ddot{\|}$ on $X$ defines a $\star$-metric $d^{\star}$ on $X$ which is given by $d^{\star}(x, y)=\ddot{\|} x \ominus y \| \ddot{\|},(x, y \in X)$, and is called the $\star$-metric induced by the $\star$-norm.

Let $z^{\star} \in \mathbb{C}^{\star}$ be an arbitrary element. The distance function $d^{\star}\left(z^{\star}, \theta^{\star}\right)$ is called $\star$-norm of $z^{\star}$. In other words,

$$
\begin{aligned}
\ddot{\|} z^{\star} \ddot{\|} & =d^{\star}\left(z^{\star}, \theta^{\star}\right)=\left(\iota(\dot{a}-\dot{0})^{2_{\alpha}} \ddot{+}(\ddot{b}-\ddot{0})^{2_{\beta}}\right)^{(1 / 2)_{\beta}} \\
& =\beta\left\{\sqrt{a^{2}+b^{2}}\right\},
\end{aligned}
$$

where $z^{\star}=(\dot{a}, \ddot{b})$ and $\theta^{\star}=(\dot{0}, \ddot{0})$.

In particular, in multiplicative calculus by taking $\alpha=I$, the identity function and $\beta=\exp$, the exponential function, and the axioms of $\star$-normed space turn into

$$
\begin{aligned}
& (\mathrm{N}(\mathrm{MC}) 1) \ddot{\|} x \ddot{\|}=1 \Leftrightarrow x=\theta^{\star}\left(\theta^{\star}=(0,1)\right), \\
& (\mathrm{N}(\mathrm{MC}) 2) \ddot{\|} \lambda \odot x \ddot{\|}=\ddot{\|} x \ddot{\|}^{|\lambda|}, \\
& (\mathrm{N}(\mathrm{MC}) 3) \ddot{\|} x \oplus y \ddot{\|} \leq \ddot{\|} x \ddot{\|} \ddot{\|} y \ddot{\|} .
\end{aligned}
$$

Then we say that $(X, \ddot{\|} \cdot \ddot{\|})$ is multiplicative normed space.
Definition 8 (see [21]). Let $z^{\star}=(\dot{a}, \ddot{b}) \in \mathbb{C}^{\star}$. We define the $\star$-complex conjugate $\bar{z}^{\star}$ of $z^{\star}$ by $\bar{z}^{\star}=\left(\alpha\{a\}, \beta\left\{-\beta^{-1}(\ddot{b})\right\}\right)=$ $(\dot{a}, \ddot{-} \ddot{b})$. Conjugation changes the sign of the imaginary part of $z^{\star}$ but leaves the real part the same. Thus

$$
\begin{aligned}
& \operatorname{Re}\left(\bar{z}^{\star}\right)=\operatorname{Re}\left(z^{\star}\right)=\left(z^{\star} \oplus \bar{z}^{\star}\right) \dot{j}=\dot{a}, \\
& \operatorname{Im}\left(\bar{z}^{\star}\right)=\ddot{-} \operatorname{Im}\left(z^{\star}\right)=\left(z^{\star} \ominus \bar{z}^{\star}\right) \ddot{/} \ddot{2}=\ddot{b} .
\end{aligned}
$$

Remark 9 (see [21]). The following conditions hold.

(i) Let $z_{1}^{\star}=\left(\dot{a}_{1}, \ddot{b}_{1}\right), z_{2}^{\star}=\left(\dot{a}_{2}, \ddot{b}_{2}\right) \in \mathbb{C}^{\star}$. We can give the $\star$-division of two $\star$-complex numbers $z_{1}^{\star}$ and $z_{2}^{\star}$ as

$$
\begin{aligned}
& z_{1}^{\star} \oslash z_{2}^{\star} \\
& \quad=\left(\alpha\left\{\frac{\left(a_{1} a_{2}+b_{1} b_{2}\right)}{\left(a_{2}^{2}+b_{2}^{2}\right)}\right\}, \beta\left\{\frac{\left(b_{1} a_{2}-a_{1} b_{2}\right)}{\left(a_{2}^{2}+b_{2}^{2}\right)}\right\}\right) .
\end{aligned}
$$

(ii) Let $\alpha$ and $\beta$ be the same generators and let $z^{\star}=$ $(\dot{a}, \ddot{b}) \in \mathbb{C}^{\star}$. Then, the relation $z^{\star} \odot \overline{z^{\star}}=\ddot{\|} z^{\star} \ddot{\|}^{2_{\beta}}$ holds. Really,

$$
\begin{aligned}
& z^{\star} \odot \overline{z^{\star}}=(\dot{a}, \ddot{b}) \odot(\dot{a}, \ddot{-} \ddot{b})=\left(\alpha\left\{a^{2}+b^{2}\right\}, \beta(0)\right) \\
& =\beta\left\{a^{2}+b^{2}\right\}=\beta\left\{\left(\beta^{-1} \beta \sqrt{a^{2}+b^{2}}\right)^{2}\right\} \\
& =\ddot{\|} z^{\star} \ddot{\|}^{2_{\beta}} \text {. }
\end{aligned}
$$

Theorem 10 (see [19]). ( $\left.\mathbb{C}^{\star}, d^{\star}\right)$ is a complete metric space, where $d^{\star}$ is defined by (13).

Corollary 11 (see [19]). $\mathbb{C}^{\star}$ is a Banach space with the $\star$-norm $\ddot{\|} \cdot \ddot{\|}$ defined by $\ddot{\|} z^{\star} \ddot{\|}=\left(\iota(\dot{a})^{2 \alpha} \ddot{+} \ddot{b}^{2_{\beta}}\right)^{(1 / 2)_{\beta}} ; z^{\star}=(\dot{a}, \ddot{b}) \epsilon$ $\mathbb{C}^{\star}$.

Following Tekin and Başar [19], we can give some examples of $\star$-normed sequence spaces. First, consider the following relations which are derived from the corresponding metrics given in (13) by putting, as usual, $\ddot{\|} z^{\star} \| \ddot{\|}=d^{\star}\left(z^{\star}, \theta^{\star}\right)$.

Theorem 12 (see [19]). The following statements hold.

(a) The spaces $\ell_{\infty}^{\star}, c^{\star}$, and $c_{0}^{\star}$ are Banach spaces with the norm $\|\cdot\|_{\infty}^{\star}$ defined by

$\|z\|_{\infty}^{\star}:=\sup _{k \in \mathbb{N}} \ddot{\|} z_{k}^{\star} \ddot{\|} ; \quad z=\left(z_{k}^{\star}\right) \in \lambda^{\star}, \lambda \in\left\{\ell_{\infty}, c, c_{0}\right\}$.

(b) The space $\ell_{p}^{\star}$ is Banach spaces with the norm $\|\cdot\|_{p}^{\star}$ defined by

$$
\|z\|_{p}^{\star}:=\left(\sum_{k} \ddot{\|} z_{k}^{\star} \ddot{\|}^{p_{\beta}}\right)^{(1 / p)_{\beta}} ; \quad p \geq 1, z=\left(z_{k}^{\star}\right) \in \ell_{p}^{\star} .
$$


Theorem 13 (see [20]). (a) The spaces $b s^{\star}, c s^{\star}$, and $c s_{0}^{\star}$ are Banach spaces with the norm $\|\cdot\|_{b s}^{*}$ defined by

$$
\begin{aligned}
\|x\|_{b s}^{\star}:=\|x\|_{c s}^{\star}:=\sup _{n \in \mathbb{N}} \ddot{\|} \sum_{*} \sum_{k=0}^{n} x_{k} \ddot{\|} ; \\
x=\left(x_{k}\right) \in \mu^{\star}, \mu \in\left\{b s, c s, c s_{0}\right\} .
\end{aligned}
$$

(b) The spaces $b v^{\star}, b v_{p}^{\star}(p \geq 1)$, and $b v_{\infty}^{\star}$ are Banach spaces with the corresponding norms defined by

$$
\begin{aligned}
\|x\|_{b v}^{\star} & :=\sum_{k} \ddot{\|}\left(\Delta^{\prime} x\right)_{k} \ddot{\|}, \\
\|x\|_{b v_{p}}^{\star} & :=\left(\sum_{k} \ddot{\|}(\Delta x)_{k} \ddot{\|}^{p_{\beta}}\right)^{(1 / p)_{\beta}}, \\
\|x\|_{b v_{\infty}}^{\star} & :=\sup _{k \in \mathbb{N}} \ddot{\|}(\Delta x)_{k} \ddot{\|},
\end{aligned}
$$

where $\left(\Delta^{\prime} x\right)_{k}=\left(x_{k} \ominus x_{k+1}\right)$ and $(\Delta x)_{k}=\left(x_{k} \ominus x_{k-1}\right), x_{-1}=\theta^{\star}$ for all $k \in \mathbb{N}$.

Analogous to classical analysis, a sequence space $\mu^{\star}$ with a linear $\star$-metric topology (cf. [19]) is called a ${ }^{\star} K$-space provided that each of the maps $p_{i}: \mu^{\star} \rightarrow \mathbb{C}^{\star}$ defined by $p_{i}(x)=x_{i}$ is $\star$-continuous by (9) for all $i \in \mathbb{N}$. Additionally, a ${ }^{\star} K$-space $\mu^{\star}$ is called an ${ }^{\star} F K$-space provided that $\mu^{\star}$ is a complete linear non-Newtonian metric space, denoted by *-linear (see [20]). An ${ }^{\star}$ FK-space whose non-Newtonian topology is normable and is called a ${ }^{\star} \mathrm{BK}$-space.

\section{Some Inequalities and Inclusion Relations}

Definition 14 (Schauder basis). If a $\star$-normed sequence space $\lambda^{\star}$ contains a sequence $\left(b_{n}\right)$ with the property that for every $x \in \lambda^{\star}$ there is a unique sequence of scalars $\left(\xi_{n}\right)$ such that

$$
{ }^{\star} \lim _{n \rightarrow \infty}\left\|x \ominus\left(\xi_{0} \odot b_{0} \oplus \xi_{1} \odot b_{1} \oplus \cdots \oplus \xi_{n} \odot b_{n}\right)\right\|^{\star}=\theta^{\star}
$$

with corresponding norm, then $\left(b_{n}\right)$ is called a Schauder basis (in non-Newtonian sense) briefly $\star$-basis, for $\lambda^{\star}$. The series ${ }_{\star} \sum_{k} \xi_{k} \odot b_{k}$ which has the sum $x$ is then called the expansion of $x$ with respect to $\left(b_{n}\right)$ and is written as $x={ }_{\star} \sum_{k} \xi_{k} \odot b_{k}$. The concepts of Schauder and algebraic $\star$-bases coincide for finite dimensional spaces. Nevertheless, there are $\star$-linear spaces without a Schauder $\star$-basis.

Let $e=\left(e_{k}\right)$ and $e^{(n)}=\left(e_{k}^{(n)}\right),(n \in \mathbb{N})$ be the sequences with $e_{k}=1^{\star}$ for all $k \in \mathbb{N}$, and $e_{k}^{(n)}=\delta_{n k}^{\star}$, where $\delta_{n k}^{\star}$ denotes the non-Newtonian Kronecker delta defined by

$$
\delta_{n k}^{\star}= \begin{cases}1^{\star}, & n=k, \\ \theta^{\star}, & n \neq k .\end{cases}
$$

Example 15. The sequence $\left\{e, e^{(0)}, e^{(1)}, \ldots\right\}_{k \in \mathbb{N}}$ is a Schauder $\star$-basis for the space $c^{\star}$ and any $x=\left(x_{k}\right)$ in $c^{\star}$ has a unique representation of the form

$$
\begin{aligned}
x=\xi \odot e \oplus * \sum_{k}\left(x_{k} \ominus \xi\right) \odot e^{(k)}, & \\
& \text { where }{ }^{\star} \lim _{k \rightarrow \infty} x_{k}=\xi .
\end{aligned}
$$

Theorem 16. The space bs ${ }^{\star}$ is norm isomorphic to the space $\ell_{\infty}^{\star}$; that is, bs $\cong \ell_{\infty}^{\star}$.

Proof. To prove this, we should show the existence of a $\star$ norm preserving linear bijection between the spaces $b s^{\star}$ and $\ell_{\infty}^{\star}$.

Consider the transformation $T$ defined from $b s^{\star}$ to $\ell_{\infty}^{\star}$ by $T x=\left({ }_{\star} \sum_{j=0}^{k} x_{j}\right)$. By using the corresponding operations $\oplus$ and $\odot$, the $\star$-linearity of $T$ is obvious. Further, it is trivial that $x=\theta^{\star}$ whenever $T x=\theta^{\star}$ and hence $T$ is injective. Let $y=$ $\left(y_{k}\right) \in \ell_{\infty}^{\star}$ and define the sequence $x=\left(x_{k}\right)$ by $x_{k}=y_{k} \ominus y_{k-1}$ for all $k \in \mathbb{N}$ with $y_{-1}=\theta^{\star}$. Then, we obtain that

$$
\begin{aligned}
\sup _{k \in \mathbb{N}} \ddot{\|} \sum_{j=0}^{k} x_{j} \ddot{\|} & =\sup _{k \in \mathbb{N}} \ddot{\|} \sum_{j=0}^{k}\left(y_{j} \ominus y_{j-1}\right) \ddot{\|}=\sup _{k \in \mathbb{N}} \ddot{\|} y_{k} \ddot{\|} \\
& =\|y\|_{\infty}^{\star}<\infty .
\end{aligned}
$$

Thus, we observe that $\|x\|_{b s}^{\star}<\infty$ and hence $x \in b s^{\star}$. Consequently $T$ is surjective and is norm preserving. Hence, $T$ is a linear bijection which therefore says that the spaces $b s^{\star}$ and $\ell_{\infty}^{\star}$ are norm isomorphic, as desired.

Theorem 17. Then the following relations are satisfied.

(i) $\mu \subseteq \mu^{\star}$ holds for each $\mu \in\left\{\ell_{\infty}, c, c_{0}, \ell_{p}, b s, c s, c s_{0}\right.$, $\left.b v, b v_{\infty}\right\}$.

(ii) $\ell_{1}^{\star} \subseteq c s^{\star} \subseteq c_{0}^{\star} \subseteq c^{\star} \subseteq \ell_{\infty}^{\star} \subseteq \omega^{\star}$ and $\ell_{1}^{\star} \subseteq b v_{0}^{\star} \subseteq b v^{\star} \subseteq$ $c^{\star}$, where $b v_{0}^{\star}:=b v^{\star} \cap c_{0}^{\star}$.

(iii) If the inverse function $\beta^{-1}$ is bounded in classical mean, then $c_{0} \subseteq c_{0}^{\star} \subseteq c \subseteq c^{\star} \subseteq \ell_{\infty} \subseteq \ell_{\infty}^{\star} \subseteq \omega$ holds.

Proof. Since the proof is trivial for the conditions (i) and (ii), we prove only (iii).

(iii) Using (i) and (ii) we need only to show $\ell_{\infty}^{\star} \subseteq \omega, c^{\star} \subseteq$ $\ell_{\infty}$, and $c_{0}^{\star} \subseteq c$. Now, consider $z=\left(z_{k}\right) \in c^{\star}$ is given. Then for every $\varepsilon \ddot{>} \ddot{0}$ there exist an $n_{0}=n_{0}(\varepsilon) \in \mathbb{N}$ and $l \in \mathbb{C}^{\star}$ such that $d^{\star}\left(z_{k}, \ell\right) \ddot{<} \varepsilon$ for all $n>n_{0}$. Since $\beta^{-1}$ is a bounded function there exists an element $M>0$ such that $\left|\beta^{-1}(x)\right|<M$ for all $x \in \mathbb{R}$. On the other hand, by applying the well-known inequality

$$
\ddot{\|} z_{k} \ddot{\|} \ddot{\leq} \ddot{\|} z_{k} \ominus \ell \ddot{\|} \ddot{+} \| \ell \ddot{\|} \ddot{\leq} \epsilon \ddot{+} \ddot{\|} \ell \ddot{\|}
$$

which implies that $\left|z_{k}\right|=\beta^{-1} \ddot{\|} z_{k} \| \leq \beta^{-1}(\epsilon \ddot{+} \ddot{\|} \ell \ddot{\|})$. Therefore, by taking into account the boundedness of $\beta^{-1}$ there exists $M_{0}>0$ such that $\left|\beta^{-1}(\epsilon \ddot{+} \ddot{\|} \ell \ddot{\|})\right|<M_{0}$; we obtain that $\left(z_{k}\right)$ is bounded in classical mean. Thus $z \in$ $\ell_{\infty}$. Hence $c^{\star} \subseteq \ell_{\infty}$. The remaining part can be obtained similarly. 
Corollary 18. The spaces $\ell_{\infty}^{\star}, c^{\star}, c_{0}^{\star}, b s^{\star}, c s^{\star}, b v_{p}^{\star}$, and $\ell_{p}^{\star}$ are $\star$-norm isomorphic to the spaces $\ell_{\infty}, c, c_{0}, b s, c s, b v_{p}$, and $\ell_{p}$, respectively.

Now, we give some well-known inequalities in the nonNewtonian sense which are essential in the study.

Lemma 19 (Young's inequality). Let $p$ and $q$ be conjugate real numbers. Then,

$$
u \dot{\times} v \leq \frac{u^{p_{\alpha}}}{\dot{p}} \cdot \dot{+} \frac{v^{q_{\alpha}}}{\dot{q}}
$$

holds for all $u, v \in \mathbb{R}_{\alpha}^{+}$and $p>1$.

Proof. For any generator function $\alpha$, we must show that the following inequality holds:

$$
\alpha^{-1}(u) \alpha^{-1}(v) \leq \frac{\left(\alpha^{-1}(u)\right)^{p}}{p}+\frac{\left(\alpha^{-1}(v)\right)^{q}}{q} .
$$

It is trivial that (29) holds for $u=\dot{0}$ or $v=\dot{0}$. Let $u, v$ be nonzero $\alpha$-real numbers. Consider the function $f:[0, \infty] \subseteq$ $\mathbb{R}_{\alpha} \rightarrow \mathbb{R}_{\beta} \subseteq \mathbb{R}$ defined by

$$
f(t)=(\iota(t))^{\lambda_{\beta}} \ddot{-} \ddot{\lambda} \ddot{x}_{\iota}(t)=\beta\left\{\left(\alpha^{-1}(t)\right)^{\lambda}-\lambda \alpha^{-1}(t)\right\},
$$

where $\iota=\beta \circ \alpha^{-1}$ and $0<\lambda<1$. Then, the $\star$-derivative of $f$ (see [23]) can be written as

$$
\begin{aligned}
f^{\star}(t) & =\beta\left\{\frac{\left(\beta^{-1} \circ f\right)^{\prime}(t)}{\left(\alpha^{-1}\right)^{\prime}(t)}\right\} \\
& =\beta\left\{\frac{\left(\left(\alpha^{-1}\right)^{\lambda}-\lambda \alpha^{-1}\right)^{\prime}(t)}{\left(\alpha^{-1}\right)^{\prime}(t)}\right\} \\
& =\beta\left\{\lambda\left(\alpha^{-1}(t)\right)^{\lambda-1}-\lambda\right\} .
\end{aligned}
$$

From the first derivative test in non-Newtonian sense, the condition $f^{\star}(t)=\ddot{0}$ holds and $t=\dot{i}$ is a critial point of $f$. Besides this,

$$
\begin{aligned}
f^{\star \star}(t) & =\beta\left\{\frac{\left(\beta^{-1} f^{\star}(t)\right)^{\prime}}{\left(\alpha^{-1}\right)^{\prime}(t)}\right\} \\
& =\beta\left\{\lambda(\lambda-1)\left(\alpha^{-1}(t)\right)^{\lambda}\right\}
\end{aligned}
$$

and, by using the second derivative test in non-Newtonian sense we have $f^{\star \star}(i)=\beta\{\lambda(\lambda-1)\} \ddot{<} \ddot{0}$ which implies that $f$ has a maximum at $i$; that is, $f(i)=\beta\{1-\lambda\}$. In other words, we say that

$$
\left[\alpha^{-1}(t)\right]^{\lambda}-\lambda \alpha^{-1}(t) \leq 1-\lambda \quad \forall t \in[0, \infty] \subseteq \mathbb{R}_{\alpha} .
$$

Now taking $t=(u)^{p_{\alpha}} j(v)^{q_{\alpha}}=\alpha\left\{\left(\alpha^{-1}(u)\right)^{p} /\left(\alpha^{-1}(v)\right)^{q}\right\}$ and $\lambda=1 / p$ in (33), we get

$$
\begin{aligned}
& \left(\frac{\left(\alpha^{-1}(u)\right)^{p}}{\left(\alpha^{-1}(v)\right)^{q}}\right)^{1 / p}-\frac{1}{p} \frac{\left(\alpha^{-1}(u)\right)^{p}}{\left(\alpha^{-1}(v)\right)^{q}} \leq 1-\frac{1}{p}, \\
& \frac{\alpha^{-1}(u)}{\alpha^{-1}(v)^{q / p}}\left(\alpha^{-1}(v)\right)^{q} \\
& \leq\left(1-\frac{1}{p}+\frac{1}{p} \frac{\alpha^{-1}(u)^{p}}{\alpha^{-1}(v)^{q}}\right)\left(\alpha^{-1}(v)\right)^{q} .
\end{aligned}
$$

Hence the inclusion (29) holds. This step completes the proof.

Theorem 20 (Hölder's inequality). Let $p$ and $q$ be conjugate positive real numbers and $u_{k}^{\star}, v_{k}^{\star} \in \mathbb{C}^{\star}$ for $k \in\{0,1,2, \ldots, n\}$. Then, the following inequality holds:

$$
\begin{aligned}
\beta \sum_{k=0}^{n} \ddot{\|} u_{k}^{\star} \odot v_{k}^{\star} \ddot{\|} \ddot{\leq}\left(\beta \sum_{k=0}^{n} \ddot{\|} u_{k}^{\star} \ddot{\|^{p_{\beta}}}\right)^{(1 / p)_{\beta}} \\
\ddot{\times}\left(\beta \sum_{k=0}^{n} \ddot{\|} v_{k}^{\star} \ddot{\|}^{q_{\beta}}\right)^{(1 / q)_{\beta}} .
\end{aligned}
$$

Proof. The inequality clearly holds when $u=\theta^{\star}=(\dot{0}, \ddot{0})$ or $v=(\dot{0}, \ddot{0})$. We may assume $u, v \neq \theta^{\star}$ in the following proof. Let

$$
\begin{aligned}
& \varepsilon=\left(\beta \sum_{k=0}^{n} \ddot{\|} u_{k}^{\star} \ddot{\|}^{p_{\beta}}\right)^{(1 / p)_{\beta}}, \\
& \delta=\left(\sum_{k=0}^{n} \ddot{\|} v_{k}^{\star} \ddot{\|}^{q_{\beta}}\right)^{(1 / q)_{\beta}}
\end{aligned}
$$

and, $s_{k}^{\star}=u_{k}^{\star} \oslash \varepsilon, t_{k}^{\star}=v_{k}^{\star} \oslash \delta$ where $\varepsilon=(\dot{0}, \ddot{\varepsilon}) \in \mathbb{R}_{\beta} \subseteq \mathbb{C}^{\star}$ and $\delta=(\dot{0}, \ddot{\delta}) \in \mathbb{R}_{\beta} \subseteq \mathbb{C}^{*}$. By taking into account Lemma 19 for each $k \in\{0,1,2, \ldots, n\}$, we obtain

$$
\ddot{\|} s_{k}^{\star} \odot t_{k}^{\star} \ddot{\|}=\ddot{\|} s_{k}^{\star} \ddot{\|} \ddot{x} \ddot{\|} t_{k}^{\star} \ddot{\|} \ddot{\leq} \frac{\ddot{\|} s_{k}^{\star} \ddot{\|}^{p_{\beta}}}{\ddot{p}}: \ddot{+} \frac{\ddot{\|} t_{k}^{\star} \ddot{\|}^{q_{\beta}}}{\ddot{q}} \text { : }
$$

which implies that

$$
\beta \sum_{k=0}^{n} \ddot{\|} s_{k}^{\star} \odot t_{k}^{\star} \ddot{\|} \ddot{s}_{\beta} \sum_{k=0}^{n} \frac{\ddot{\|} s_{k}^{\star} \ddot{\|}^{p_{\beta}}}{\ddot{p}}: \ddot{+}_{\beta} \sum_{k=0}^{n} \frac{\ddot{\|} t_{k}^{\star} \ddot{\|}^{q_{\beta}}}{\ddot{q}}: .
$$

Then, as is easy to see,

$$
\begin{aligned}
& \beta \sum_{k=0}^{n} \ddot{\|}\left(u_{k}^{\star} \oslash \varepsilon\right) \odot\left(v_{k}^{\star} \oslash \delta\right) \ddot{\|} \\
& \quad \ddot{\leq}_{\beta} \sum_{k=0}^{n} \frac{\ddot{\|} u_{k}^{\star} \ddot{\|}^{p_{\beta}}}{\varepsilon \ddot{x} \ddot{p}}: \ddot{+}_{\beta} \sum_{k=0}^{n} \frac{\ddot{\|} v_{k}^{\star} \ddot{\|}^{q_{\beta}}}{\delta \ddot{x} \ddot{q}}:=\frac{\ddot{1}}{\ddot{p}} \ddot{+} \frac{\ddot{1}}{\ddot{q}}=\ddot{1} .
\end{aligned}
$$

Therefore, we deduce by combining this with the inclusion (39) that (35) holds for every $k \in\{0,1,2, \ldots, n\}$. 
In particular, for $p=2$ the inequality (35) turns out to be

$$
\beta \sum_{k=0}^{n} \ddot{\|} u_{k}^{\star} \odot v_{k}^{\star} \ddot{\|} \ddot{\leq}\left(\beta \sum_{k=0}^{n} \ddot{\|} u_{k}^{\star} \ddot{\|}^{2_{\beta}} \ddot{x}_{\beta} \sum_{k=0}^{n} \ddot{\|} v_{k}^{\star} \ddot{\|}^{2_{\beta}}\right)^{(1 / 2)_{\beta}}
$$

denoted by Cauchy-Schwartz inequality in non-Newtonian sense.

Theorem 21 (Minkowski's inequality). Let $p \geq 1$ and $u_{k}^{\star}, v_{k}^{\star} \in$ $\mathbb{C}^{\star}$ for all $k \in\{0,1,2, \ldots, n\}$. Then,

$$
\begin{aligned}
& \left(\beta \sum_{k=0}^{n} \ddot{\|} u_{k}^{\star} \oplus v_{k}^{\star} \ddot{\|}^{p_{\beta}}\right)^{(1 / p)_{\beta}} \\
& \ddot{\leq}\left(\beta \sum_{k=0}^{n} \ddot{\|} u_{k}^{\star} \ddot{\|}^{p_{\beta}}\right)^{(1 / p)_{\beta}} \ddot{+}\left(\beta \sum_{k=0}^{n} \ddot{\|} v_{k}^{\star} \ddot{\|}^{p_{\beta}}\right)^{(1 / p)_{\beta}} .
\end{aligned}
$$

Proof. The case $p=1$ is trivial. Let $p>1$ and $u_{k}^{\star}, v_{k}^{\star} \in \mathbb{C}^{\star}$. One can immediately conclude that

$$
\begin{aligned}
\beta \sum_{k=0}^{n} \ddot{\|} u_{k}^{\star} \oplus v_{k}^{\star} \ddot{\|}^{p_{\beta}} \ddot{\leq} \sum_{k=0}^{n} \ddot{\|} u_{k}^{\star} \ddot{\|} \ddot{x} \ddot{\|} u_{k}^{\star} \oplus v_{k}^{\star} \ddot{\|}^{(p-1)_{\beta}} \\
\ddot{+} \beta \sum_{k=0}^{n} \ddot{\|} v_{k}^{\star} \ddot{\|} \ddot{x} \ddot{\|} u_{k}^{\star} \oplus v_{k}^{\star} \ddot{\|}^{(p-1)_{\beta}} .
\end{aligned}
$$

This leads us with Theorem 20 to the consequence that

$$
\begin{aligned}
\beta \sum_{k=0}^{n} \ddot{\|} u_{k}^{\star} \oplus v_{k}^{\star} \ddot{\|} p_{\beta} \\
\ddot{\leq}\left[\left(\beta \sum_{k=0}^{n} \ddot{\|} u_{k}^{\star} \ddot{\|}\right)^{p_{\beta}} \ddot{(1 / p)_{\beta}} \ddot{+}\left(\beta \sum_{k=0}^{n} \ddot{\|} v_{k}^{\star} \ddot{\|}^{p_{\beta}}\right)^{(1 / p)_{\beta}}\right] \\
\ddot{\times}\left(\beta \sum_{k=0}^{n} \ddot{\|} u_{k}^{\star} \oplus v_{k}^{\star} \ddot{\|}^{(q p-q)_{\beta}}\right)^{(1 / q)_{\beta}} .
\end{aligned}
$$

This concludes the proof.

\section{Non-Newtonian Paranormed Sequence Spaces}

Firstly, we give the definition of non-Newtonian paranorm, briefly $\star$-paranorm.

Definition 22. Let $X$ be a real or complex $\star$-linear space and let $g^{\star}$ be a subadditive function from $X$ to the subset $\mathbb{R}_{\beta}^{+} \subseteq$ $\mathbb{R}^{+}$. Then the pair $\left(X, g^{\star}\right)$ is called a $\star$-paranormed space and $g^{\star}$ is a $\star$-paranorm, for $X$, if the following axioms are satisfied for all elements $x, y \in X$ and for all scalars $\lambda$.

$(\mathrm{N}(\mathrm{PN}) 1) \quad g^{\star}(x)=\ddot{0}$ if $x=\theta^{\star},\left(\theta^{\star}=\left(0^{\star}, 0^{\star}, \ldots\right)\right)$.

$(\mathrm{N}(\mathrm{PN}) 2) \quad g^{\star}(\ominus x)=g^{\star}(x),(\ominus x$ is opposite $\star$-vector of $x)$.

$(\mathrm{N}(\mathrm{PN}) 3) \quad g^{\star}(x \oplus y) \ddot{\leq} g^{\star}(x) \ddot{+} g^{\star}(y)$.
( $\mathrm{N}(\mathrm{PN}) 4)$ If $\left(\lambda_{n}^{\star}\right)$ is a sequence of complex scalars, that is, $\lambda^{\star}=(\dot{\lambda}, \ddot{\lambda})$ with $\lambda_{n}^{\star} \stackrel{d^{\star}}{\longrightarrow} \lambda^{\star}$ as $n \rightarrow \infty$ and $x_{n}, x \in X$ for all $n \in \mathbb{N}$ with $x_{n} \stackrel{g^{\star}}{\longrightarrow} x$, then $\lambda_{n}^{\star} \odot x_{n} \stackrel{g^{\star}}{\longrightarrow} \lambda^{\star} \odot x$ as $n \rightarrow \infty$.

In particular, in bigeometric calculus case, that is, $\alpha=\beta=$ exp, the conditions (N(PN)1), (N(PN)2), and $(\mathrm{N}(\mathrm{PN}) 4)$ also hold with zero $\star$-vector $\theta^{\star}=((1,1),(1,1), \ldots)$, and $(\mathrm{N}(\mathrm{PN}) 3)$ turns into

$(\mathrm{BG}(\mathrm{PN}) 3) \quad g^{\star}(x \oplus y) \leq g^{\star}(x) g^{\star}(y)$.

Assume hereafter that $p=\left(p_{k}\right)$ is a bounded sequence of strictly positive real numbers, so that $0<p_{k} \leq \sup p_{k}=$ $H<\infty$ and $M=\max \{1, H\}$. We will assume throughout that $p_{k} \times p_{k}^{\prime}=p_{k}+p_{k}^{\prime}$ provided that $1<\inf p_{k} \leq H<\infty$ for all $k \in \mathbb{N}$.

Quite recently Tekin and Başar [19] have introduced the sets $\ell_{\infty}^{\star}, c^{\star}, c_{0}^{\star}$, and $\ell_{p}^{\star}$ of sequences over the complex field $\mathbb{C}^{\star}$ which correspond to the sets $\ell_{\infty}, c, c_{0}$, and $\ell_{p}$ over the complex field $\mathbb{C}$, respectively. It is natural to expect that the Banach spaces $\ell_{\infty}^{\star}, c^{\star}, c_{0}^{\star}$, and $\ell_{p}^{\star}$ can be extended to the complete $\star$-paranormed sequence spaces so as the Maddox's spaces are derived on the real or complex field from the classical sequence spaces. Now, we may give the spaces $\ell_{\infty}^{\star}(p), c^{\star}(p), c_{0}^{\star}(p), \ell^{\star}(p), b s^{\star}(p), c s^{\star}(p), c s_{0}^{\star}(p), b v^{\star}(p)$, and $b v_{\infty}^{\star}(p)$ in non-Newtonian sense which correspond to the well-known examples of the paranormed sequence spaces in (CC):

$$
\begin{aligned}
& \ell_{\infty}^{\star}(p):=\left\{x=\left(x_{k}\right) \in \omega^{\star}: \sup _{k \in \mathbb{N}} \ddot{\|} x_{k} \ddot{\|}^{\left(p_{k}\right)_{\beta}} \ddot{<} \infty\right\}, \\
& :=\left\{x=\left(x_{k}\right)=\left(\dot{\varepsilon}_{k}, \ddot{\delta}_{k}\right) \in \omega^{\star}: \sup _{k \in \mathbb{N}} \beta\left\{\sqrt{\varepsilon_{k}^{2}+\delta_{k}^{2}} p_{k}\right\}\right. \\
& <\infty\} \\
& c^{\star}(p):=\left\{x=\left(x_{k}\right) \in \omega^{\star}: \exists l \in \mathbb{C}^{\star}\right. \\
& \left.\ni^{\star} \lim _{k \rightarrow \infty} \ddot{\|} x_{k} \ominus l \ddot{\|}^{\left(p_{k}\right)_{\beta}}=\theta^{\star}\right\} \\
& :=\left\{x=\left(x_{k}\right)=\left(\dot{\varepsilon}_{k}, \ddot{\delta}_{k}\right) \in \omega^{\star}: \exists l=(\dot{\ell}, \ddot{\ell}) \in \mathbb{C}^{\star}\right. \\
& \left.\ni^{\beta} \lim _{k \rightarrow \infty} \beta\left\{\left(\ell \sqrt{2\left(\varepsilon_{k}^{2}+\delta_{k}^{2}\right)}\right)^{p_{k}}\right\}=\ddot{0}\right\}, \\
& c_{0}^{\star}(p)=\left\{x=\left(x_{k}\right) \in \omega^{\star}:{ }^{\star} \lim _{k \rightarrow \infty} \ddot{\|} x_{k} \ddot{\|}^{\left(p_{k}\right)_{\beta}}=\theta^{\star}\right\} \text {, } \\
& :=\left\{x=\left(x_{k}\right)=\left(\dot{\varepsilon}_{k}, \ddot{\delta}_{k}\right) \in \omega^{\star}:{ }^{\beta} \lim _{k \rightarrow \infty} \beta\left\{\sqrt{\varepsilon_{k}^{2}+\delta_{k}^{2}} p_{k}\right\}\right. \\
& <\infty\},
\end{aligned}
$$




$$
\begin{aligned}
& \ell^{\star}(p)=\left\{x=\left(x_{k}\right) \in \omega^{\star}: \beta \sum_{k} \ddot{\|} x_{k} \ddot{\|}^{\left(p_{k}\right)_{\beta}} \ddot{<}, \quad\left(0<p_{k}<\infty\right),\right. \\
& :=\left\{x=\left(x_{k}\right)=\left(\dot{\varepsilon}_{k}, \ddot{\delta}_{k}\right) \in \omega^{\star}: \beta\left\{\sum_{k} \sqrt{\varepsilon_{k}^{2}+\delta_{k}^{2}} p_{k}\right\}\right. \\
& \quad<\infty\} .
\end{aligned}
$$

Following Kadak [20], we define the several sets $b s^{\star}(p)$, $c s^{\star}(p), c s_{0}^{\star}(p), b v^{\star}(p)$, and $b v_{\infty}^{\star}(p)$ of sequences in the sense of non-Newtonian calculus as follows:

$$
\begin{aligned}
& b s^{\star}(p):=\left\{x=\left(x_{k}\right) \in \omega^{\star}:\left(\sum_{j=0}^{k} x_{j}\right) \in \ell_{\infty}^{\star}(p)\right\}, \\
& :=\left\{x=\left(x_{k}\right)=\left(\dot{\varepsilon}_{k}, \ddot{\delta}_{k}\right) \in \omega^{\star}:\right. \\
& \left.\sup _{k \in \mathbb{N}}\left\{\sqrt{\left(\sum_{j=0}^{k} \varepsilon_{j}\right)^{2}+\left(\sum_{j=0}^{k} \delta_{j}\right)^{2}}\right\}<\infty\right\}, \\
& c s^{\star}(p):=\left\{x=\left(x_{k}\right) \in \omega^{\star}:\left(\sum_{j=0}^{k} x_{j}\right) \in c^{\star}(p)\right\}, \\
& :=\left\{x=\left(x_{k}\right)=\left(\dot{\varepsilon}_{k}, \ddot{\delta}_{k}\right) \in \omega^{\star}:\right. \\
& \lim _{k \rightarrow \infty} \beta\left\{\sqrt{\left(\sum_{j=0}^{k} \varepsilon_{j}-\ell\right)^{2}+\left(\sum_{j=0}^{k} \delta_{j}-\ell\right)^{2}}\right\} \\
& <\infty\}, \quad\left(\ell=(\dot{\ell}, \ddot{\ell}) \in \mathbb{C}^{\star}\right), \\
& c s_{0}^{\star}(p):=\left\{x=\left(x_{k}\right) \in \omega^{\star}:\left(\star \sum_{k=0}^{n} x_{k}\right) \in c_{0}^{\star}(p)\right\}, \\
& b v^{\star}(p)=\left\{x=\left(x_{k}\right) \in \omega^{\star}: \beta \sum_{k=0}^{\infty} \ddot{\|} \Delta x_{k} \ddot{\|}^{\left(p_{k}\right)_{\beta}}<\infty\right\} \text {, } \\
& \left(\Delta x_{k}=x_{k} \ominus x_{k-1} ; x_{-1}=\theta^{\star}\right) \text {, }
\end{aligned}
$$

$$
\begin{aligned}
&:=\left\{x=\left(x_{k}\right)=\left(\dot{\varepsilon}_{k}, \ddot{\delta}_{k}\right) \in \omega^{\star}:\right. \\
&\left.\beta\left\{\sum_{k=0}^{\infty}{\sqrt{\left(\varepsilon_{k}-\varepsilon_{k-1}\right)^{2}+\left(\delta_{k}-\delta_{k-1}\right)^{2}}}^{p_{k}}\right\}<\infty\right\}, \\
& b v_{\infty}^{\star}(p):=\left\{x=\left(x_{k}\right) \in \omega^{\star}: \sup _{k \in \mathbb{N}} \ddot{\|} \Delta x_{k} \ddot{\|}^{\left(p_{k}\right)_{\beta}}<\infty\right\} .
\end{aligned}
$$

It is a routine verification that each of the sets $\ell_{\infty}^{\star}(p), c^{\star}(p)$, $c_{0}^{\star}(p), \ell^{\star}(p), b s^{\star}(p), c s^{\star}(p), c s_{0}^{\star}(p), b v^{\star}(p)$, and $b v_{\infty}^{\star}(p)$ is a $\star$-linear space.

Theorem 23. The following statements hold.

(i) Define the functions $g^{\star}$ and $\tilde{g}^{\star}$ by

$$
\begin{aligned}
& g^{\star}(x)=\sup _{k \in \mathbb{N}} \ddot{\|} x_{k} \ddot{\|}^{\left(p_{k} / M\right)_{\beta}}, \\
& \tilde{g}^{\star}(x)=\left(\beta \sum_{k} \ddot{\|} x_{k} \ddot{\|}^{\left(p_{k}\right)_{\beta}}\right)^{(1 / M)_{\beta}} .
\end{aligned}
$$

Then $c^{\star}(p)$ and $c_{0}^{\star}(p)$ are complete $\star$-paranormed spaces by $g^{\star}$ if $p_{k} \in \ell_{\infty}^{\star}$. Also the spaces $\ell_{\infty}^{\star}(p)$ and $\ell^{\star}(p)$ are complete $\star$-paranormed spaces paranormed by $g^{\star}$ and $\tilde{g}^{\star}$, respectively if and only if inf $p_{k}>0$.

(ii) The sets $b s^{\star}(p), c s^{\star}(p)$, and $c s_{0}^{\star}(p)$ of sequences are the complete $\star$-paranormed spaces paranormed by $g_{1}^{\star}$ by

$$
g_{1}^{\star}(x)=\sup _{k \in \mathbb{N}} \ddot{\|} \star \sum_{j=0}^{k} x_{j} \ddot{\|}^{\left(p_{k} / M\right)_{\beta}} \quad \text { iff inf } p_{k}>0 \text {. }
$$

(iii) The sets $b v^{\star}(p)$ and $b v_{\infty}^{\star}(p)$ are the complete $\star-$ paranormed spaces by $g_{2}^{\star}$ and $g_{3}^{\star}$ defined by

$$
\begin{aligned}
& g_{2}^{\star}(x)=\left(\beta \sum_{k} \ddot{\|} \Delta x_{k} \ddot{\|}^{\left(p_{k}\right)_{\beta}}\right)^{(1 / M)_{\beta}}, \\
& g_{3}^{\star}(x)=\sup _{k \in \mathbb{N}} \ddot{\|} \Delta x_{k} \ddot{\|}^{(1 / M)_{\beta}} \text { iff inf } p_{k}>0,
\end{aligned}
$$

respectively, where $\Delta x_{k}=x_{k} \ominus x_{k-1} ; x_{-1}=\theta^{\star}$ for all $k \in \mathbb{N}$.

Proof. To avoid repetition of similar statements, we give the proof only for the space $b v^{\star}(p)$ in case (iii). The remaining parts can be obtained similarly.

The $\star$-linearity of $b v^{\star}(p)$ with respect to coordinatewise addition and scalar multiplication follows from the following 
inequalities which are satisfied for $u, x \in b v^{\star}(p)$ (see Theorem 21):

$$
\begin{gathered}
\left(\star \sum_{k} \ddot{\|} \Delta\left(u_{k} \oplus x_{k}\right) \ddot{\|}^{\left(p_{k}\right)_{\beta}}\right)^{(1 / M)_{\beta}} \\
\ddot{\leq}\left(\sum_{k} \ddot{\|} \Delta u_{k} \ddot{\|}^{\left(p_{k}\right)_{\beta}}\right)^{(1 / M)_{\beta}} \\
\ddot{+}\left(* \sum_{k} \ddot{\|} \Delta x_{k} \ddot{\|}^{\left(p_{k}\right)_{\beta}}\right)^{(1 / M)_{\beta}}
\end{gathered}
$$

and the condition

$$
\ddot{\|} \lambda^{\star} \ddot{\|}^{\left(p_{k}\right)_{\beta}} \ddot{\leq} \max \left\{\ddot{1}, \ddot{\|} \lambda^{*} \ddot{\|}^{M_{\beta}}\right\}
$$

holds for any scalar $\lambda^{\star}=(\dot{\lambda}, \ddot{\lambda}) \in \mathbb{C}^{\star}$ (cf. [3]). It is clear that $g^{\star}\left(\theta^{\star}\right)=\ddot{0}$ and $g^{\star}(\ominus x)=g^{\star}(x)$ for all $x \in b v^{\star}(p)$. Hence, by combining the inclusions (49) and (50) with subadditivity of $g^{\star}$ we get the inequality $g^{\star}\left(\lambda^{\star} \odot\right.$ $x) \ddot{\leq} \max \left\{\ddot{1}, \ddot{\|} \lambda^{\star} \ddot{\|}\right\} \ddot{\times} g^{\star}(x)$.

Let $\left(x^{n}\right)$ be any sequence of the points of the space $b v^{\star}(p)$ such that $g^{\star}\left(x^{n} \ominus x\right) \rightarrow \theta^{\star}$ and let $\left(\lambda_{n}\right)$ be any sequence of $\star$-complex scalars such that $\lambda_{n}^{\star} \rightarrow \lambda^{\star}$ with corresponding $\star$-metric. Then, since the $\star$-triangle inequality $g^{\star}\left(x^{n}\right) \ddot{\leq} g^{\star}(x) \ddot{+} g^{\star}\left(x^{n} \ominus x\right)$ holds, the sequence $\left\{g^{\star}\left(x^{n}\right)\right\}$ is $\beta$-bounded and we thus have

$$
\begin{aligned}
g^{\star} & \left(\lambda_{n}^{\star} \odot x^{n} \ominus \lambda^{\star} \odot x\right) \\
& =\left(\beta \sum_{k} \ddot{\|} \Delta\left(\lambda_{n}^{\star} \odot x_{k}^{(n)} \ominus \lambda^{\star} \odot x_{k}\right) \ddot{\|}{ }^{\left(p_{k}\right)_{\beta}}\right)^{(1 / M)_{\beta}} \\
& \ddot{\leq} \ddot{\|} \lambda_{n}^{\star} \ominus \lambda^{\star} \ddot{\|} \ddot{\times} g^{\star}\left(x^{n}\right) \ddot{+} \ddot{\|} \lambda^{\star} \ddot{\|} \ddot{\times} g^{\star}\left(x^{n} \ominus x\right),
\end{aligned}
$$

which tends to $\ddot{0}$ as $n \rightarrow \infty$. That is to say that the scalar multiplication is $\star$-continuous. Hence, $g^{\star}$ is a $\star$-paranorm on the space $b v^{\star}(p)$.

It remains to prove the $\star$-completeness of the space $b v^{\star}(p)$. Let $\left\{x^{i}\right\}$ be any Cauchy sequence in the space $b v^{\star}(p)$, where $x_{i}=\left\{x_{0}^{(i)}, x_{1}^{(i)}, x_{2}^{(i)}, \ldots\right\}$. Then, for a given $\epsilon \ddot{>} \ddot{0}$ there exists a positive integer $n_{0}(\epsilon)$ such that

$$
g^{\star}\left(x^{i} \ominus x^{j}\right) \ddot{<} \ddot{\epsilon} \quad \forall i, j \geq n_{0}(\epsilon) .
$$

By taking into account the definition of $g^{\star}$ for each fixed $k \epsilon$ $\mathbb{N}$, we have that

$$
\begin{aligned}
\ddot{\|} & \Delta\left[\left(x^{i}\right)_{k} \ominus\left(x^{j}\right)_{k}\right] \ddot{\|} \\
& \ddot{\leq}\left(\beta \sum_{k} \ddot{\|} \Delta\left[\left(x^{i}\right)_{k} \ominus\left(x^{j}\right)_{k}\right] \ddot{\|}^{\left(p_{k}\right)_{\beta}}\right)^{(1 / M)_{\beta}} \ddot{<} \epsilon
\end{aligned}
$$

$$
\forall i, j \geq n_{0}(\epsilon),
$$

which leads us to the fact that $\left\{\left(\Delta x^{0}\right)_{k},\left(\Delta x^{1}\right)_{k},\left(\Delta x^{2}\right)_{k}, \ldots\right\}$ is a Cauchy sequence for every fixed $k \in \mathbb{N}$. Since $\mathbb{C}^{\star}$ is complete (see [19]), it $\star$-converges; that is, $\left(\Delta x^{i}\right)_{k} \rightarrow x_{k}$ as $i \rightarrow \infty$. Using these infinitely many limits $x_{0}, x_{1}, x_{2}, \ldots$, we define the sequence $\left\{x_{0}, x_{1}, x_{2}, \ldots\right\}$. From the inclusion (52) for each $m \in \mathbb{N}$ and $i, j \geq n_{0}(\epsilon)$ we have

$$
\beta \sum_{k=0}^{m} \ddot{\|} \Delta\left[\left(x^{i}\right)_{k} \ominus\left(x^{j}\right)_{k}\right] \ddot{\|}^{\left(p_{k}\right)_{\beta}} \ddot{\leq} g^{\star}\left(x^{i} \ominus x^{j}\right)^{M_{\beta}} \ddot{<} \epsilon^{M_{\beta}} .
$$

Take any $i \geq n_{0}(\epsilon)$. First let $j \rightarrow \infty$ in (54) and then $m \rightarrow \infty$, to obtain $g^{\star}\left(x^{i} \ominus x\right) \ddot{\leq} \epsilon$. We have by Minkowski's inequality for each $m \in \mathbb{N}$ that

$$
\begin{aligned}
& \left(\sum_{k} \ddot{\|}(\Delta x)_{k} \ddot{\|}^{\left(p_{k}\right)_{\beta}}\right)^{(1 / M)_{\beta}} \ddot{\leq} g^{\star}\left(x^{i} \ominus x\right) \ddot{+} g^{\star}\left(x^{i}\right) \\
& \quad \ddot{\epsilon} \epsilon \ddot{+} g^{\star}\left(x^{i}\right),
\end{aligned}
$$

which implies that $x \in b v^{\star}(p)$. Since $g^{\star}\left(x^{i} \ominus x\right) \ddot{\leq} \epsilon$ for all $i \geq n_{0}(\epsilon)$ it follows that $x^{i} \rightarrow x$ as $i \rightarrow \infty$. Therefore, we have shown that $b v^{\star}(p)$ is $\star$-complete.

It is trivial to show that the $\star$-paranormed spaces $\ell_{\infty}^{\star}(p), c^{\star}(p), c_{0}^{\star}(p), \ell^{\star}(p), b s^{\star}(p), c s^{\star}(p), c s_{0}^{\star}(p), b v^{\star}(p)$, and $b v_{\infty}^{\star}(p)$ may be reduced to some new sequence spaces in the special cases of the sequences $\left(p_{k}\right)$ and generator functions. For instance, the sequence space $b v^{\star}(p)$ corresponds in the case $p_{k}=p$ for all $k \in \mathbb{N}$ to the sequence space $b v_{p}^{\star}$ of $p$ bounded variation sequences in [20].

Now, as a consequence of Corollary 18, the following corollary presents the relations between $\star$-paranormed and classic paranormed spaces.

Corollary 24. The following statements hold.

(i) The space $\mu^{\star}(p)$ is norm isomorphic to the usual paranormed space $\mu(p)$ where $\mu \in\left\{\ell_{\infty}, c, c_{0}, \ell_{p}, b s, c s\right.$, $\left.c s_{0}, b v, b v_{\infty}\right\}$.

(ii) $\mu(p) \subseteq \mu^{\star}(p)$ and $\ell_{1}^{\star}(p) \subseteq c s^{\star}(p) \subseteq c_{0}^{\star}(p) \subseteq c^{\star}(p) \subseteq$ $\ell_{\infty}^{\star}(p) \subseteq \omega^{\star}$.

4.1. Duality Properties. Following [24], we give the alpha-, beta-, and gamma-duals of a $\star$-paranormed sequence space $\lambda^{\star}(p) \subset \omega^{\star}$ which are, respectively, denoted by $\left\{\lambda^{\star}(p)\right\}^{\alpha}$, $\left\{\lambda^{\star}(p)\right\}^{\beta}$, and $\left\{\lambda^{\star}(p)\right\}^{\gamma}$ as follows:

$$
\begin{aligned}
& \left\{\lambda^{\star}(p)\right\}^{\alpha}:=\left\{w=\left(w_{k}\right) \in \omega^{\star}: w \odot z=\left(w_{k} \odot z_{k}\right)\right. \\
& \left.\quad \in \ell_{1}^{\star}(p) \forall z=\left(z_{k}\right) \in \lambda^{\star}(p)\right\}, \\
& \left\{\lambda^{\star}(p)\right\}^{\beta}:=\left\{w=\left(w_{k}\right) \in \omega^{\star}: w \odot z=\left(w_{k} \odot z_{k}\right)\right. \\
& \left.\quad \in c s^{\star}(p) \forall z=\left(z_{k}\right) \in \lambda^{\star}(p)\right\}, \\
& \left\{\lambda^{\star}(p)\right\}^{\gamma}:=\left\{w=\left(w_{k}\right) \in \omega^{\star}: w \odot z=\left(w_{k} \odot z_{k}\right)\right. \\
& \left.\quad \in b s^{\star}(p) \forall z=\left(z_{k}\right) \in \lambda^{\star}(p)\right\},
\end{aligned}
$$


where $\left(w_{k} \odot z_{k}\right)$ is the coordinatewise product of $\star$-complex numbers $w$ and $z$ for all $k \in \mathbb{N}$. Throughout the text, we also use the notation " $<$ " for a $\star$-linear subspace which was created in [18].

Theorem 25. Let $\emptyset \neq \lambda^{\star}(p) \subset \omega^{\star}$. Then the following statements are valid.
(a) $\left\{\lambda^{\star}(p)\right\}^{\beta}$ is a sequence space if $\left\{\lambda^{\star}(p)\right\}^{\beta} \subset \omega^{\star}$.
(b) If $\lambda^{\star}(p) \subset \mu^{\star}(p) \subset \omega^{\star}$, then $\left\{\mu^{\star}(p)\right\}^{\beta} \subset\left\{\lambda^{\star}(p)\right\}^{\beta}$.
(c) $\lambda^{\star} \subset\left\{\lambda^{\star}\right\}^{\beta \beta}:=\left(\left\{\lambda^{\star}\right\}^{\beta}\right)^{\beta}$.
(d) $\left\{c_{0}^{\star}(p)\right\}^{\beta}=\left\{c^{\star}(p)\right\}^{\beta}=\left\{\ell_{\infty}^{\star}(p)\right\}^{\beta}=\ell_{1}^{\star}(p)$.
(e) $\left\{\ell_{1}^{\star}(p)\right\}^{\beta}=\ell_{\infty}^{\star}(p)$.

Proof. Since the proofs are trivial for the conditions (b) and (c), we prove only (a), (d), and (e). Let $w=\left(w_{k}\right), m=\left(m_{k}\right)$, and $n=\left(n_{k}\right) \in\left\{\lambda^{\star}(p)\right\}^{\beta}$.

(a) It is trivial that $\left\{\lambda^{\star}(p)\right\}^{\beta} \subset \omega^{\star}$ holds from the hypothesis. We show that $m \oplus n \in\left\{\lambda^{\star}(p)\right\}^{\beta}$ for $m, n \in$ $\left\{\lambda^{\star}(p)\right\}^{\beta}$. Suppose that $l \in \lambda^{\star}(p)$. Then $\left(m_{k} \odot l_{k}\right) \in$ $c s^{\star}(p)$ and $\left(n_{k} \odot l_{k}\right) \in c s^{\star}(p)$ for all $l \in \lambda^{\star}(p)$. We can deduce that

$$
\begin{aligned}
&\left(\left(m_{k} \oplus n_{k}\right) \odot l_{k}\right)=\left(m_{k} \odot l_{k}\right) \oplus\left(n_{k} \odot l_{k}\right) \in c s^{\star}(p) \\
& \forall l \in \lambda^{\star}(p) .
\end{aligned}
$$

Hence, $m \oplus n \in\left\{\lambda^{\star}(p)\right\}^{\beta}$. Now, we show that $t \odot w \in$ $\left\{\lambda^{\star}(p)\right\}^{\beta}$ for any $t \in \mathbb{C}^{\star}$ and $w=\left(w_{k}\right) \in\left\{\lambda^{\star}(p)\right\}^{\beta}$. Since $\left(w_{k} \odot l_{k}\right) \in c s^{\star}(p)$ for all $l \in \lambda^{\star}(p)$ and combining this with $\left(\left(t_{k} \odot w_{k}\right) \odot l_{k}\right)=t_{k} \odot\left(w_{k} \odot l_{k}\right) \epsilon$ $c s^{\star}(p)$ for all $l \in \lambda^{\star}(p)$, we get $t \odot w \in\left\{\lambda^{\star}(p)\right\}^{\beta}$. Therefore, we have proved that $\left\{\lambda^{\star}(p)\right\}^{\beta}$ is a subspace of the space $\omega^{\star}$.

(d) Obviously $\left\{\ell_{\infty}^{\star}(p)\right\}^{\beta} \subset\left\{c^{\star}(p)\right\}^{\beta} \subset\left\{c_{0}^{\star}(p)\right\}^{\beta}$ by Theorem 25(b). Then we must show that $\ell_{1}^{\star}(p) \subset$ $\left\{\ell_{\infty}^{\star}(p)\right\}^{\beta}$ and $\left\{c_{0}^{\star}(p)\right\}^{\beta} \subset \ell_{1}^{\star}$. Now, consider $w=$ $\left(w_{k}\right) \in \ell_{1}^{\star}(p)$ and $z=\left(z_{k}\right) \in \ell_{\infty}^{\star}(p)$ are given. By taking into account the cases ((a)-(b)) of Theorem 12, we have

$$
\begin{aligned}
\star \sum_{k}\left\|w_{k} \odot z_{k}\right\|^{\left(p_{k}\right)_{\beta}} \stackrel{\ddot{\leq}}{\sup _{k \in \mathbb{N}} \ddot{\|} z_{k} \ddot{\|}^{(1 / M)_{\beta}}} \\
\quad \ddot{\times}\left(\sum_{k} \ddot{\|} w_{k} \ddot{\|}^{\left(p_{k}\right)_{\beta}}\right)^{(1 / M)_{\beta}} \\
\ddot{\leq} g^{\star}(z) \ddot{\times} \tilde{g}^{\star}(w)<\infty,
\end{aligned}
$$

where $\sup p_{k} \leq 1$ which implies that $w \odot z \in c s^{\star}(p)$. So the condition $\ell_{1}^{\star}(p) \subset\left\{\ell_{\infty}^{\star}(p)\right\}^{\beta}$ holds.

Conversely, for a given $y=\left(y_{k}\right) \in \omega^{\star} \backslash \ell_{1}^{\star}(p)$ we prove the existence of an $x \in c_{0}^{\star}(p)$ with $y \odot x \notin c s^{\star}(p)$. According to $y \notin \ell_{1}^{\star}(p)$ we can choose an index sequence $\left(n_{r}\right)$ which is strictly increasing with $n_{0}=0$ and $\sum_{k=n_{r-1}}^{n_{r}-1} \ddot{\|} y_{k} \|^{\left(p_{k}\right)_{\beta}} \ddot{>} r ;(r \in \mathbb{N})$. By taking into account Remark 9(i) we define $x=\left(x_{k}\right) \in c_{0}^{\star}(p)$ by $x_{k}:=\left(\operatorname{sgn}^{\star} y_{k} \oslash r\right)$ where the $\star$-complex signum function is defined by

$$
\operatorname{sgn}^{\star}(y):= \begin{cases}\bar{y} \oslash \ddot{\|} y \ddot{\|}, & y \neq \theta^{\star}, \\ \theta^{\star}, & y=\theta^{\star}\end{cases}
$$

for all $y=\left(y_{k}\right) \in \mathbb{C}^{\star}$. Finally, by using Remark 9(ii) and taking the generators $\alpha=\beta$ we get

$$
\begin{aligned}
& \star \sum_{k=n_{r-1}}^{n_{r}-1}\left(y_{k} \odot x_{k}\right)^{\left(p_{k}\right)_{\beta}} \\
& \quad=\star \sum_{k=n_{r-1}}^{n_{r}-1}\left[y_{k} \odot\left(\operatorname{sgn}^{\star} y_{k} \oslash r\right)\right]^{\left(p_{k}\right)_{\beta}} \\
& =\frac{1}{r^{\left(p_{k}\right)_{\beta}}} \odot \star \sum_{k=n_{r-1}}^{n_{r}-1} \ddot{\|} y_{k} \ddot{\|}^{\left(p_{k}\right)_{\beta}} \geq \ddot{1}, \quad\left(\sup p_{k} \leq 1\right) .
\end{aligned}
$$

Therefore, $y \odot x \notin c s^{\star}(p)$ and thus $y \notin\left\{c_{0}^{\star}(p)\right\}^{\beta}$. Hence $\left\{c_{0}^{\star}(p)\right\}^{\beta} \subset \ell_{1}^{\star}(p)$. The other part of this case can be obtained similarly.

(e) From the condition (c) we have $\ell_{\infty}^{\star}(p) \quad c$ $\left(\left\{\ell_{\infty}^{\star}(p)\right\}^{\beta}\right)^{\beta}=\left\{\ell_{1}^{\star}(p)\right\}^{\beta}$ since $\left\{\ell_{\infty}^{\star}(p)\right\}^{\beta}=\ell_{1}^{\star}(p)$. Now we assume the existence of a $w=\left(w_{n}\right) \epsilon$ $\left\{\ell_{1}^{\star}(p)\right\}^{\beta} \backslash \ell_{\infty}^{\star}(p)$. Since $w$ is an $\star$-unbounded there exists a subsequence $\left(w_{n_{k}}\right)$ of $\left(w_{n}\right)$ and we can find a number $(k+1)^{2_{\beta}}$ such that $\ddot{\|} w_{n_{k}} \ddot{\|}{ }^{\left(p_{k}\right)_{\beta}} \ddot{\geq}(k+1)^{2_{\beta}}$ for all $k \in \mathbb{N}_{1}$. The sequence $\left(x_{n}\right)$ is defined by $x_{n}:=\left(\operatorname{sgn}^{\star}\left(w_{n_{k}}\right) \oslash(k+1)^{2_{\beta}}\right)$ if $n=n_{k}$, and $\theta^{\star}$ otherwise. Then $x \in \ell_{1}^{\star}(p)$. However,

$$
\star \sum_{n}\left(w_{n} \odot x_{n}\right)^{\left(p_{k}\right)_{\beta}}=\sum_{k} \frac{\ddot{\|} w_{n_{k}} \ddot{\|}^{\left(p_{k}\right)_{\beta}}}{(k+1)^{\left(2 p_{k}\right)_{\beta}}}:=\infty .
$$

Hence $w \notin\left\{\ell_{1}^{\star}(p)\right\}^{\beta}$, which contradicts our assumption and $\left\{\ell_{1}^{\star}(p)\right\}^{\beta} \subset \ell_{\infty}^{\star}(p)$. This step completes the proof.

In addition to Theorem 25, we give the following corollary which is immediate consequences of the $\zeta$-duals $(\zeta \in\{\alpha$, $\beta, \gamma\})$.

Corollary 26. For each $\zeta \in\{\alpha, \beta, \gamma\}$, the following statements hold.
(a) $\left\{\lambda^{\star}(p)\right\}^{\alpha} \subset\left\{\lambda^{\star}(p)\right\}^{\beta} \subset\left\{\lambda^{\star}(p)\right\}^{\gamma} \subset \omega^{\star}$; in particular, $\left\{\lambda^{\star}(p)\right\}^{\zeta}$ is a sequence space over $\mathbb{C}^{\star}$.
(b) $\left\{\ell_{1}^{\star}(p)\right\}^{\zeta}=\ell_{\infty}^{\star}(p)$ and $\left\{\ell_{\infty}^{\star}(p)\right\}^{\zeta}=\ell_{1}^{\star}(p)$.
(c) If $\lambda^{\star}(p) \subset \mu^{\star}(p) \subset \omega^{\star}$, then $\left\{\mu^{\star}(p)\right\}^{\zeta} \subset\left\{\lambda^{\star}(p)\right\}^{\zeta}$. 


\section{Conflict of Interests}

The authors declare that there is no conflict of interests regarding the publication of this paper.

\section{Acknowledgment}

The authors record their pleasure to the anonymous referee for his/her constructive report and many helpful suggestions on the main results.

\section{References}

[1] H. Nakano, "Modulared sequence spaces," Proceedings of the Japan Academy, vol. 27, pp. 508-512, 1951.

[2] S. Simons, "The sequence spaces $l\left(p_{v}\right)$ and $m\left(p_{v}\right)$," Proceedings of the London Mathematical Society, vol. 15, pp. 422-436, 1965.

[3] I. J. Maddox, "Paranormed sequence spaces generated by infinite matrices," Mathematical Proceedings of the Cambridge Philosophical Society, vol. 64, no. 2, pp. 335-340, 1968.

[4] C. G. Lascarides, "A study of certain sequence spaces of Maddox and a generalization of a theorem of Iyer," Pacific Journal of Mathematics, vol. 38, no. 2, pp. 487-500, 1971.

[5] C. G. Lascarides and I. J. Maddox, "Matrix transformation between some classes of sequences," Proceedings of the Cambridge Philosophical Society, vol. 68, pp. 99-104, 1970.

[6] M. Mursaleen and A. K. Noman, "On the spaces of $\lambda$-convergent and bounded sequences," Thai Journal of Mathematics, vol. 8, no. 2, pp. 311-329, 2010.

[7] M. Mursaleen and S. A. Mohiuddine, "Some matrix transformations of convex and paranormed sequence spaces into the spaces of invariant means," Journal of Function Spaces and Applications, vol. 2012, Article ID 612671, 6 pages, 2012.

[8] M. Mursaleen and S. A. Mohiuddine, "Almost bounded variation of double sequences and some four dimensional summability matrices," Publicationes Mathematicae Debrecen, vol. 75, no. 3-4, pp. 495-508, 2009.

[9] M. Kirişçi and F. Başar, "Some new sequence spaces derived by the domain of generalized difference matrix," Computers \& Mathematics with Applications, vol. 60, no. 5, pp. 1299-1309, 2010.

[10] F. Başar and M. Kirişçi, "Almost convergence and generalized difference matrix," Computers \& Mathematics with Applications, vol. 61, no. 3, pp. 602-611, 2011.

[11] S. Demiriz and C. Çakan, "On some new paranormed sequence spaces," General Mathematics Notes, vol. 1, no. 2, pp. 26-42, 2010.

[12] M. Grossman and R. Katz, Non-Newtonian Calculus, Lee Press, 1978.

[13] M. Grossman, Bigeometric Calculus, Archimedes Foundation Box 240, Rockport, Mass, USA, 1983.

[14] M. Grossman, The First Nonlinear System of Differential and Integral Calculus, Mathco, 1979.

[15] A. E. Bashirov, E. M. Kurpınar, and A. Özyapıc1, "Multiplicative calculus and its applications," Journal of Mathematical Analysis and Applications, vol. 337, no. 1, pp. 36-48, 2008.

[16] A. E. Bashirov and M. Riza, "On complex multiplicative differentiation," TWMS Journal of Applied and Engineering Mathematics, vol. 1, no. 1, pp. 75-85, 2011.

[17] E. Misirli and Y. Gurefe, "Multiplicative Adams BashforthMoulton methods," Numerical Algorithms, vol. 57, no. 4, pp. 425-439, 2011.
[18] A. F. Çakmak and F. Başar, "On the classical sequence spaces and non-Newtonian calculus," Journal of Inequalities and Applications, vol. 2012, Article ID 932734, 13 pages, 2012.

[19] S. Tekin and F. Başar, "Certain sequence spaces over the nonNewtonian complex field," Abstract and Applied Analysis, vol. 2013, Article ID 739319, 11 pages, 2013.

[20] U. Kadak, "Determination of the Kothe-Toeplitz duals over the non-Newtonian complex field," The Scientific World Journal, vol. 2014, Article ID 438924, 10 pages, 2014.

[21] U. Kadak and H. Efe, "Matrix transformations between certain sequence spaces over the non-Newtonian complex field," The Scientific World Journal, vol. 2014, Article ID 705818, 12 pages, 2014.

[22] U. Kadak and H. Efe, "The construction of Hilbert spaces over the non-Newtonian field," International Journal of Analysis, vol. 2014, Article ID 746059, 10 pages, 2014.

[23] U. Kadak and M. Özlük, "Generalized Runge-Kutta method with respect to the non-Newtonian calculus," Abstract and Applied Analysis, vol. 2015, Article ID 594685, 10 pages, 2015.

[24] F. Başar, "Normed and paranormed sequence spaces," in Summability Theory and Its Applications, pp. 15-32, Bentham Science Publishers, Istanbul, Turkey, 2012. 


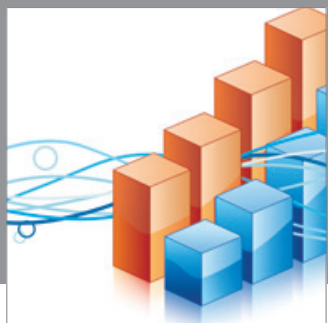

Advances in

Operations Research

mansans

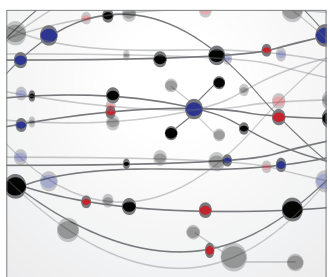

The Scientific World Journal
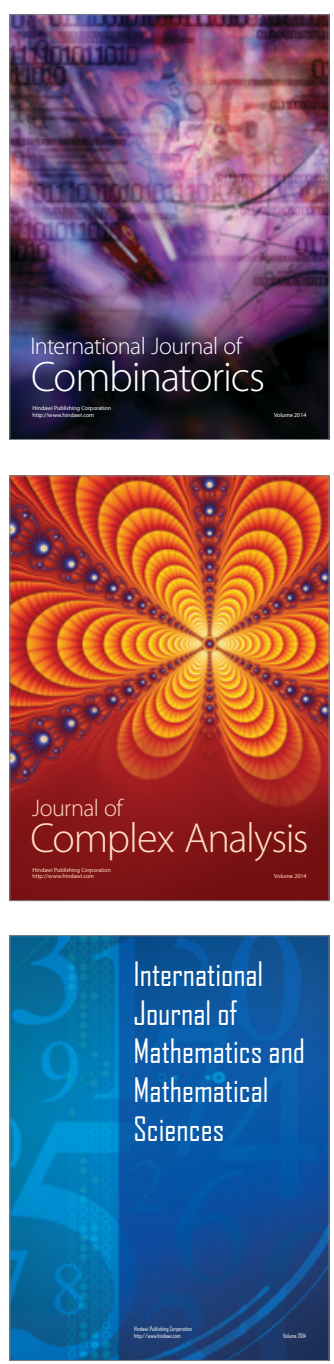
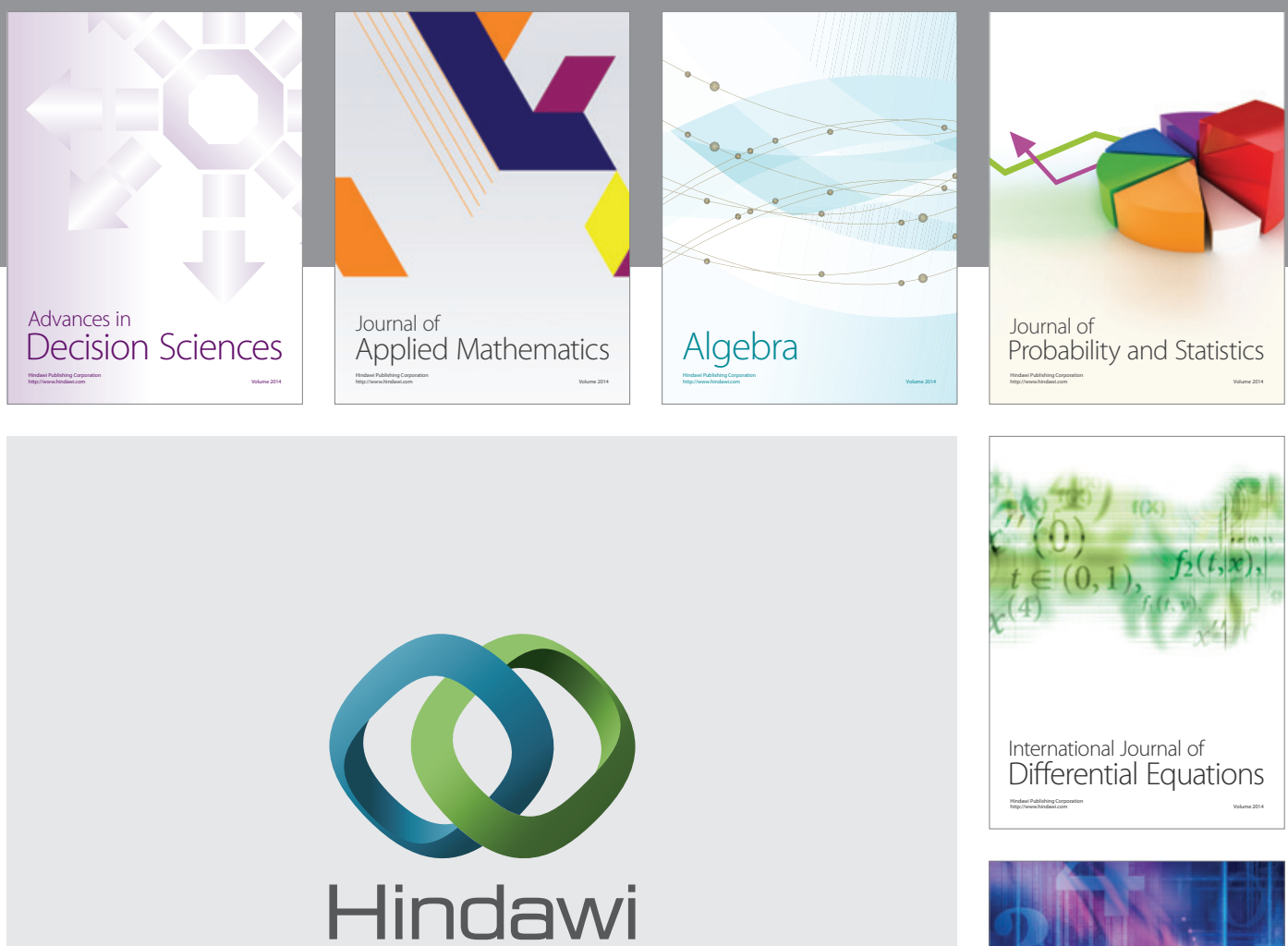

Submit your manuscripts at http://www.hindawi.com
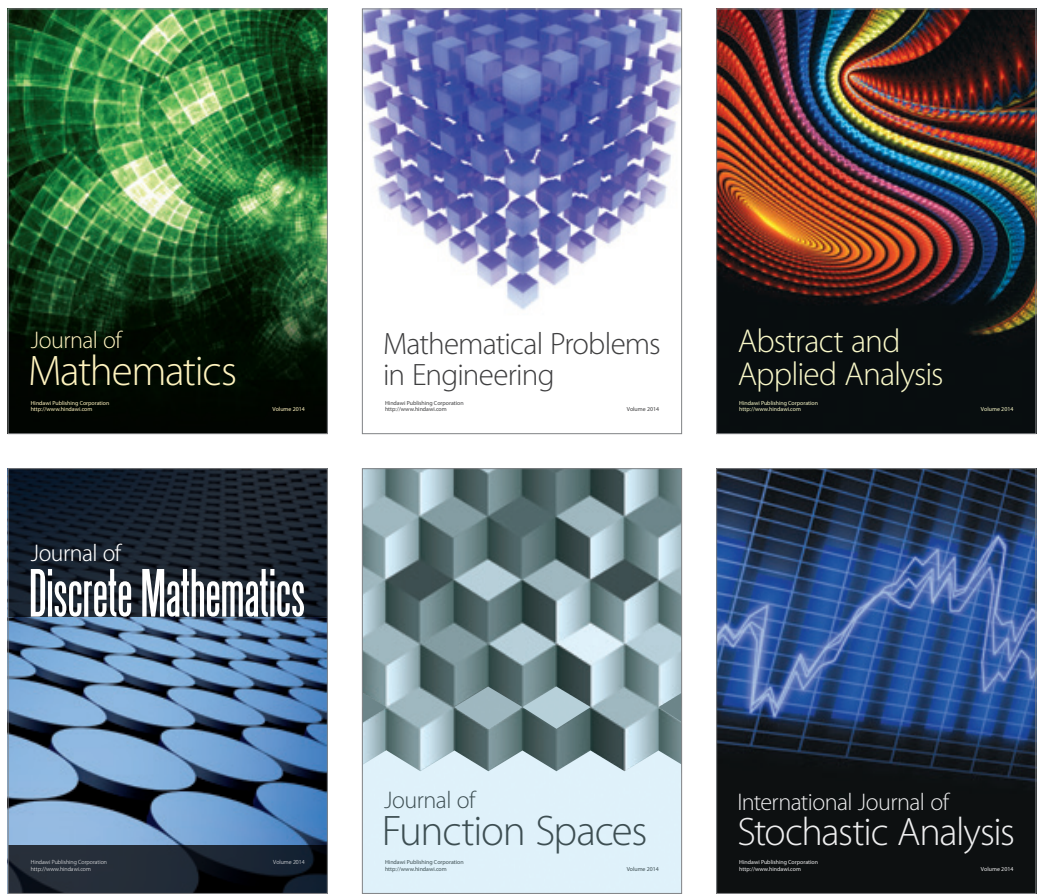

Journal of

Function Spaces

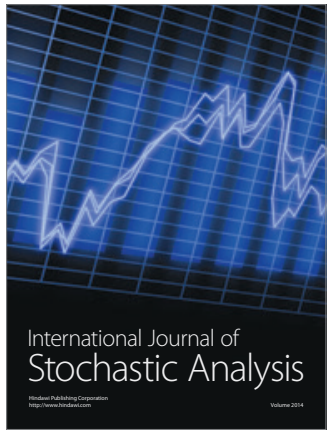

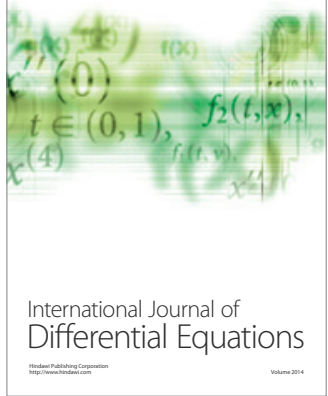
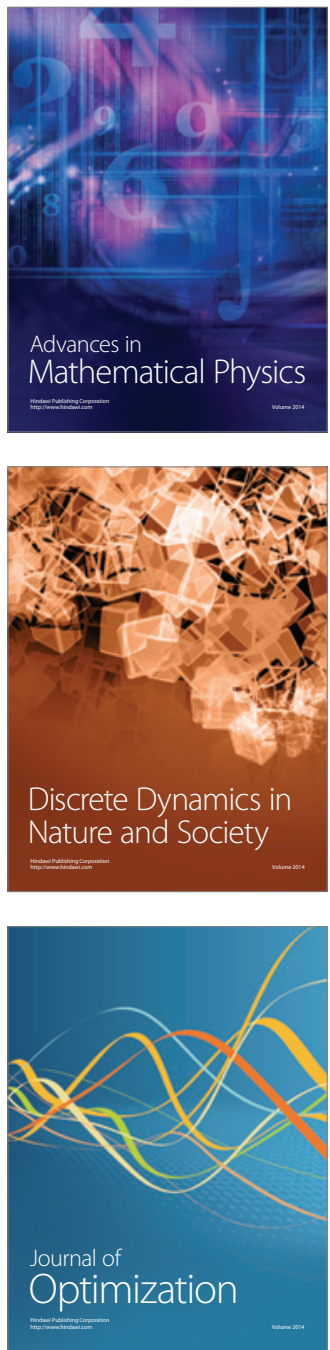